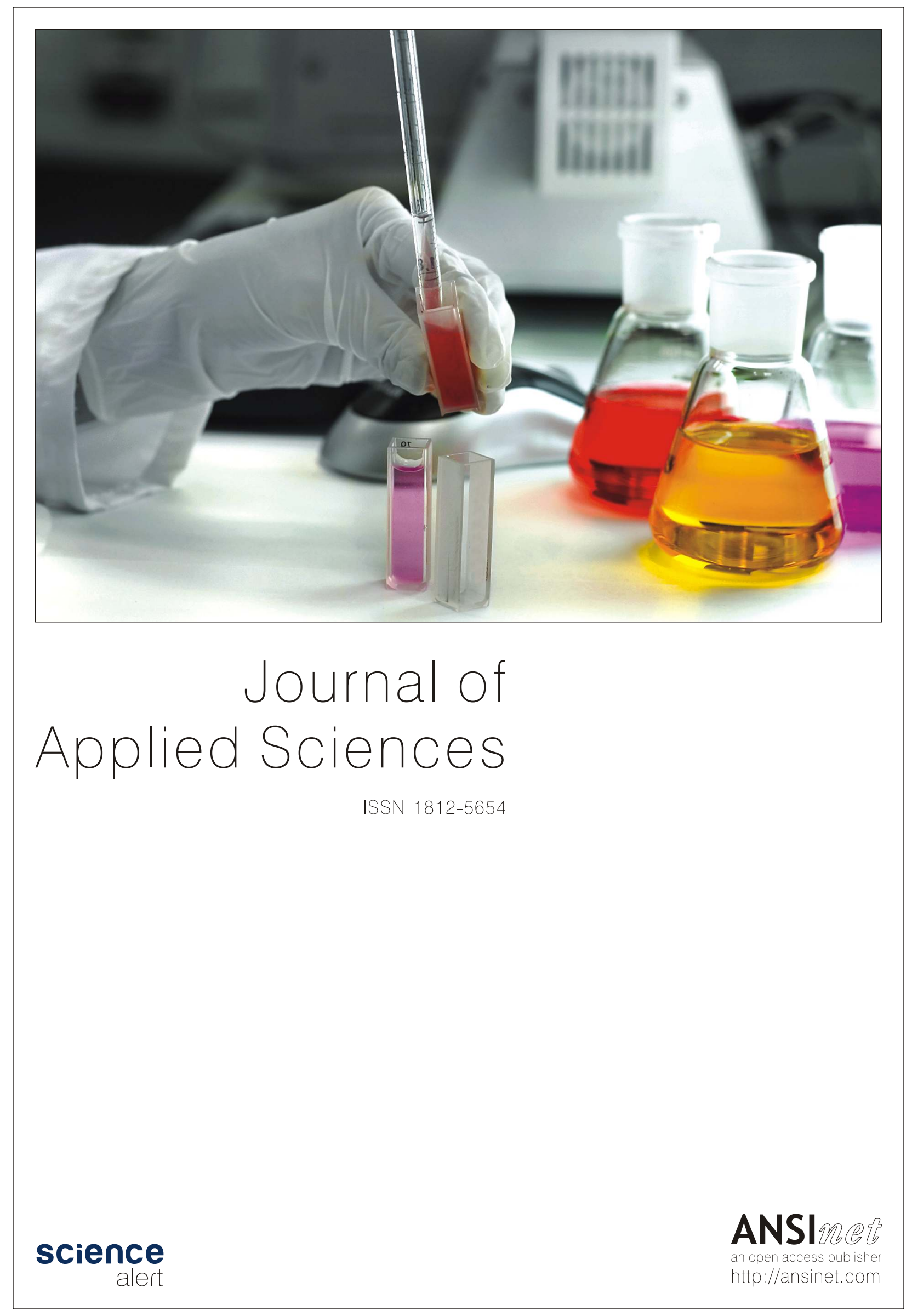


Journal of Applied Sciences 14 (1): 95-100, 2014

ISSN 1812-5654 / DOI: 10.3923/jas.2014.95.100

(C) 2014 Asian Network for Scientific Information

\title{
Tighter Receiver Performance Lower Bound for MIMO Wireless Communication Systems
}

\author{
${ }^{1}$ Muhammad Kamran Naeem, ${ }^{1}$ Mohammad Patwary, \\ ${ }^{1}$ Abdel-Hamid Soliman and ${ }^{2}$ Mohamed Abdel-Maguid \\ ${ }^{1}$ Faculty of Computing, Engineering and Sciences (FCES), Staffordshire University, Stafford, \\ ST18 0AD, United Kingdom \\ ${ }^{2}$ School of Science, Technology and Health, University Campus Suffolk (UCS), Ipswich IP4 1QJ, \\ United Kingdom
}

\begin{abstract}
Multiple transmit-receive antenna based wireless communication system is a well investigated area in the literature. However, within the scope of author's knowledge there are few analytical frameworks which facilitate communication system design engineers to select the required transmit-receive antenna combination for a given quality of service (QoS) in terms of Bit Error Rate (BER) or Symbol Error Rate (SER). Most of such frameworks for Multiple-Input Multiple-Output (MIMO) wireless systems in the existing literature are lacking tightness between theoretical approximation and actual simulation results. The aim of investigation in this study is to provide theoretical expectation of receiver performance with MIMO wireless communication system within a given channel. In this study, new analytical performance bound frameworks with tighter lower bounds have been presented for different receivers for MIMO systems. On the basis of the presented analytical framework, a simulation platform has been established. The channel state information as well as expected quality of service is assumed to be known for simplicity. Rayleigh fading channel has been considered within simulation platform. Simulation results show that the proposed frameworks provide degree of tightness up to 100 times at Signal to Noise Ratio (SNR) $20 \mathrm{~dB}$ for $(2 \times 2) \mathrm{MIMO}$ system in comparison to the existing frameworks. Few other MIMO antenna combinations have been considered in the evaluation process of the proposed framework.
\end{abstract}

Key words:MIMO systems, performance lower bound, zero forcing, minimum mean square error, maximum likelihood

\section{INTRODUCTION}

Significant amount of research has been conducted (Molisch et al., 2002; Guey et al., 1999; Alamouti, 1998; Tarokh et al., 1998; Telatar, 1999) for Multiple-Input Multiple-Output (MIMO) based communication architectures to improve the information capacity, signal detection reliability and spectral efficiency without increasing the transmit power or bandwidth as compared to single antenna systems. In MIMO systems, multiple independent transmission channels are available which increase the channel capacity as the number of antenna elements increases. It is discussed by Molisch and Win (2004) and Sanayei and Nosratinia (2004) that the robustness in terms of bit error rate and capacity of the MIMO system can be improved by exploiting antenna diversity and spatial multiplexing, respectively.

The conventional purpose of using multiple antenna combinations at both transmitter and at the receiver either to achieve transmit-receive diversity or to achieve enhanced information throughput as discussed by Zheng and Tse (2003) and Telatar (1999). Regardless of the aim, appropriate selection of detection schemes is crucial to obtain maximum achievable capacity and diversity of MIMO systems. Therefore, significant amount of research has been carried out to find detection schemes that are computationally efficient and can obtain maximum achievable diversity and capacity. With the given resources, the most commonly used detection schemes found in the existing literature are Zero Forcing (ZF), Minimum Mean Square Error (MMSE) and Maximum Likelihood (ML).

For the ease of system design to achieve a predefined capacity or quality of service requirement, analytical frameworks are extremely useful that provide a performance bench mark. Multiple antenna based future communication system is expected to be adaptive with available capacity or quality of service to offer as trade off

Corresponding Author: Muhammad Kamran Naeem, Faculty of Computing, Engineering and Sciences (FCES), Staffordshire University, Stafford, ST18 0AD, United Kingdom 
with each other. A simplified analytical framework is expected to lead towards designing such resource adaption algorithm easier. However, to achieve the effectiveness of such framework, a tighter bound is required. Most of the available lower bounds in the existing literature are lacking tightness with the actual performance. Several performance analysis frame works for ZF and MMSE detection has been presented by Tse and Viswanath (2005), Jiang et al. (2011), Matthaiou et al. (2011) and Han et al. (2010).

The contribution in this study is proposition of simplified analytical frameworks of $\mathrm{MMMO}$ receiver performance, which provides tighter lower bound in comparison to existing bounds for ZF, MMSE and ML detection schemes within $\mathrm{MMO}$ wireless communication systems. Channel state information is assumed to be known at receiver.

\section{SYSTEM MODEL}

The system model considered in this study is shown in Fig. 1. A wireless multiple input multiple output communication system with $\mathrm{N}_{t}$ number of transmit antennas is assumed with $N_{r}$ number of receiving antennas at fusion center receiver.

The signal vector $\mathrm{x}$ transmitted from $\mathrm{N}_{\mathrm{t}}$ number of transmit antennas is defined as:

$$
\mathrm{x}=\left[\mathrm{x}_{1}, \mathrm{x}_{2}, \ldots, \mathrm{x}_{\mathrm{Nt}}\right]^{\mathrm{T}}
$$

where, $x$ is with dimensions $\left(\mathrm{N}_{\mathrm{t}} \times 1\right)$. The received signal vector is expressed as:

$$
\mathrm{y}=\mathrm{Hx}+\mathrm{n}
$$

where, $\mathrm{y}$ is the received signal vector with dimensions $\left(\mathrm{N}_{\mathrm{t}} \times 1\right), \mathrm{H}$ is the rayleigh fading channel matrix of size $\left(\mathrm{N}_{\mathrm{r}} \times \mathrm{N}_{\mathrm{t}}\right)$ and $\mathrm{n}$ is the noise vector with dimensions $\left(\mathrm{N}_{\mathrm{r}} \times 1\right)$.

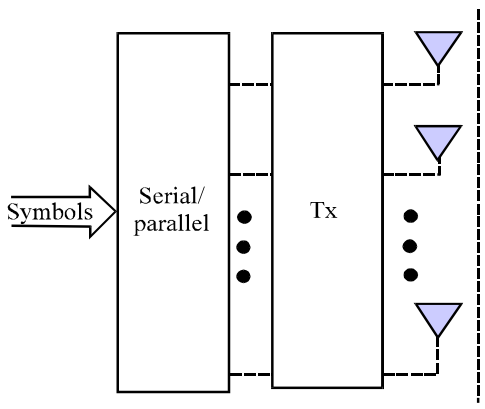

The noise is considered to be additive white Gaussian noise with zero mean and unity variance $\sigma^{2}$. The Rayleigh fading channel matrix is defined as:

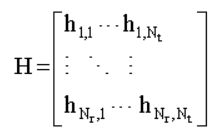

where, $h_{i, j}$ denotes the channel coefficients from ith transmitter antenna with $i \in\{1,2, \ldots, \mathrm{Nt}\}$ to $j$ th receiving antenna at the fusion center with $\mathrm{j} \in\{1,2, \ldots, \mathrm{Nr}\}$. A symmetric transmitter receiver $\mathrm{M \amalg MO}$ communication system model is considered.

\section{DETECTION METHODS UNDER-CONSIDERATION}

A brief description of the detection schemes as mentioned in introductory section of this study is provided in this section. In $\mathrm{ZF}$ detection, the received signals are send through $\mathrm{ZF}$ filter denoted as $\mathrm{G}_{\mathrm{ZF}}$ and can be defined as:

$$
\mathrm{G}_{\mathrm{ZF}}=\left(\mathrm{H}^{\mathrm{H}} \mathrm{H}\right)^{-1} \mathrm{H}^{\mathrm{H}}
$$

Subsequently, the recovered spatially multiplexed data streams recovered from the detected received signal are denoted as $\hat{\mathrm{x}}_{\mathrm{ZF}}$ and can be written as:

$$
\hat{x}_{\mathrm{ZF}}=\mathrm{G}_{\mathrm{ZF}} \mathrm{y}=\left[\left(\mathrm{H}^{\mathrm{H}} \mathrm{H}\right)^{-1} \mathrm{H}^{\mathrm{H}}\right] \mathrm{y}
$$

During the detection process, $\mathrm{ZF}$ detector is aimed to null out interfering components, which can cause noise amplification. Subsequently, it is well established that ZF is not the best possible detection scheme, although, it is simple and easy to implement. MMSE is another widely used detection scheme which provides a tradeoff between minimizing inter-symbol interference and noise amplification. MMSE filter matrix is denoted as $\mathrm{G}_{\text {MMSE }}$ and defined as:

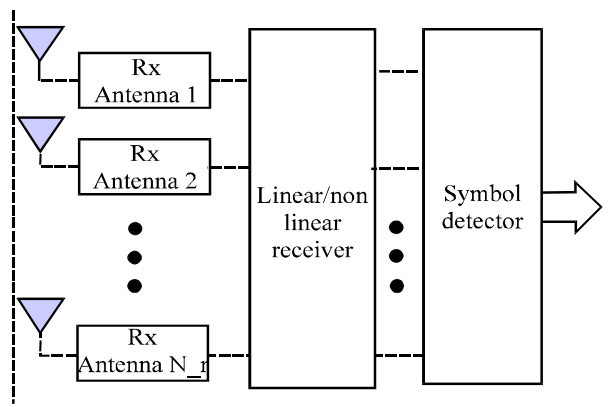

Fig. 1: Typical Multiple-Input Multiple-Output (MIMO) wireless communication system model 


$$
\mathrm{G}_{\mathrm{MMSE}}=\left(\mathrm{H}^{\mathrm{H}} \mathrm{H}+\boldsymbol{\sigma}^{2} \mathrm{I}\right)^{-1} \mathrm{H}^{\mathrm{H}}
$$

Hence, the estimation of the transmitted signal vector can be written as:

$$
\hat{\mathrm{x}}_{\text {MMSE }}=\mathrm{G}_{\text {MMSE }} \mathrm{y}=\left[\left(\mathrm{H}^{\mathrm{H}} \mathrm{H}+\mathrm{o}^{2} \mathrm{I}\right)^{-1} \mathrm{H}^{\mathrm{H}}\right] \mathrm{y}
$$

$\mathrm{ML}$ detector is known to be the optimal detector in terms of minimizing the probability of bit error rate. The criterion required to satisfy maximum likelihood detection can be defined as:

$$
\hat{\mathrm{x}}_{\mathrm{ML}}=\underset{\mathrm{kel2}: 2^{\mathrm{k}}}{\arg \min }\left\|\mathrm{y}-\mathrm{Hx}_{\mathrm{k}}\right\|^{2}
$$

where, $x_{k}$ is the $k^{\text {th }}$ candidate symbol vector out of $2^{\mathrm{N}_{t}}$ number of possible symbol vectors. However, computational complexity of these detection schemes grows exponentially with the number of antenna elements when used within MIMO systems. While designing MIMO system, selection of detection scheme along with the resources required to be provided to achieve a given quality of service is challenging. Moreover, to design adaptive receiver with power constrain, lower number of iterations are desirable to converge true performance pattern from initial approximation. One possibility of approximating the performance of these systems is to design a framework which provides tighter error performance bound.

\section{ANALYTICAL FRAMEWORK}

Bit error rate is a critical measure of system performance which defines the quality of service of any telecommunication system. An intended achievable quality of service threshold is required to be determined, to allocate resources during any given telecommunication system. To find such threshold, analytical frameworks have been studied in the literature that provide with a benchmark of required resources. The following subsection provides a brief overview of such frameworks within the existing literature, followed by the proposed analytical frameworks.

Existing framework: Most commonly used linear detection schemes e.g., ZF, MMSE and ML have been the prime topics of interest for such analytical performance measure. Recent work in Jiang et al. (2011) and Tse and Viswanath (2005) present a frame work for the analysis of error performance for ZF and MMSE detection schemes which is defined as:

$$
\begin{gathered}
\mathrm{P}_{\mathrm{b}, \mathrm{ZF}}=\left[\frac{1}{2}\left(1-\sqrt{\frac{\mathrm{snr}}{1+\mathrm{snr}}}\right)\right] \sum_{\mathrm{n}=0}^{\mathrm{N}_{\mathrm{r}}-\mathrm{N}_{\mathrm{t}}+\mathrm{H}}\left(\begin{array}{c}
\mathrm{N}_{\mathrm{r}}-\mathrm{N}_{\mathrm{r}}+\mathrm{N}+\mathrm{n} \\
\mathrm{n}
\end{array}\right)\left(\frac{1+\sqrt{\frac{\mathrm{snr}}{1+\mathrm{snr}}}}{2}\right) \\
\mathrm{P}_{\mathrm{b}, \mathrm{MMSE}}=\mathrm{E}\left[\mathrm{e}^{-n \infty, \mathrm{n}}\right] \mathrm{P}_{\mathrm{b}, \mathrm{ZF}}
\end{gathered}
$$

Where:

$$
\eta \infty, n=\left(U_{n}^{T} h_{n}\right)^{T} \Lambda^{-2}\left(U_{n}^{T} h_{n}\right)
$$

where, $U_{n}$ is the upper triangular matrix and $\Lambda$ is the eigen values matrix of $\mathrm{H}_{n}$ and $\mathrm{H}_{n}$ is the sub-matrix obtained by taking $h_{n}$ out of $H . h_{n}$ is the nth column of $H$. Equation 7 can be simplified into Eq. 11 for a symmetric MIMO system i.e.:

$$
\mathrm{P}_{\mathrm{b}, \mathrm{ZF}}=\frac{1}{2}\left(\frac{1}{1+\mathrm{snr}}\right)
$$

ML detection is widely known to be optimum in terms of bit error rate performance with the cost of intensive computational complexity. Different upper bounds on Symbol Error Rate (SER) and Bit Error Rate (BER) probability of $\mathrm{ML}$ detection within $\mathrm{MIMO}$ communication systems have been presented by Zhu and Murch (2002), Kuchi and Ayyar (2011) and Gritsch et al. (2004). The upper bounds for the probability of bit error rate defined in the existing literature are function of input signal to noise ratio and number of receive antennas. A generalized model found in the existing literature is given as follows:

$$
\mathrm{P}_{\mathrm{b}, \mathrm{NL}}=\left[\frac{1}{2}\left(1-\sqrt{\frac{\mathrm{snr}}{1+\mathrm{snr}}}\right)\right]^{\mathrm{N}_{\mathrm{r}}} \sum_{\mathrm{n}=0}^{\mathrm{N}_{\mathrm{r}}-1}\left(\begin{array}{c}
\mathrm{N}_{\mathrm{r}}-1+\mathrm{n} \\
\mathrm{n}
\end{array}\right)\left(\frac{1+\sqrt{\frac{\mathrm{snr}}{1+\mathrm{snr}}}}{2}\right)^{\mathrm{n}}
$$

As mentioned earlier, the frame work presented in Eq. 12 provides the error performance upper bound for $\mathrm{ML}$ detection. According to author's knowledge, there is no framework which provides error performance lower bound for $\mathrm{ML}$ detection without error correction code in the existing literature.

Proposed framework: For symmetric MIMO system, the existing approximated performance bounds for $\mathrm{ZF}$ and MMSE are quite loose with respect to actual simulation results. In this context, simple analytical frameworks that provide tighter lower bounds for ZF, MMSE and ML 
detection schemes are proposed in this subsection. The proposed frameworks are simple and accurate in the context of performance tightness that depends on the MIMO dimension as well as input signal to noise ratio. Denoting to be the symmetric MIMO dimension, the proposed analytical framework of bit error rate performance lower bound with $\mathrm{ZF}$ detection at the receiver can be written as:

$$
P_{b, Z F}=e^{\left(\sqrt{\frac{3}{d}}\right)} \operatorname{erfc}\left(\frac{1}{d}\right) \log _{10}(\sqrt{d})\left(\frac{1}{1+\sqrt{2 \text { snr }}}\right)
$$

The error performance bound for receiver with MMSE detection is proposed to be as in Eq. 14 which depends on input signal to noise ratio and MUMO dimension:

$$
\mathrm{P}_{\mathrm{b}, \mathrm{MMSE}}=\frac{1}{\sqrt{\mathrm{d}}} \operatorname{erfc}\left(\frac{1}{\mathrm{~d}}\right)\left(\frac{1}{2 \mathrm{snr}}\right)^{\frac{1}{4}}\left(\frac{1}{1+\sqrt{2 \mathrm{snr}}}\right)
$$

As mentioned earlier, there is no error performance lower bound framework for receiver with $\mathrm{ML}$ detection in the existing literature; a framework is proposed in Eq. 15 which defines error performance lower bound:

$$
\mathrm{P}_{\mathrm{b}, \mathrm{ML}}=\frac{\mathrm{e}^{-\sqrt{\mathrm{snn}}}}{2}\left(\frac{\sqrt{\mathrm{d}}(1+\sqrt{\mathrm{snr}})}{(\sqrt{\mathrm{d}}+\mathrm{snr})(1+\mathrm{snr})^{2}}\right)
$$

\section{SIMULATION AND RESULTS}

To evaluate the performance of the proposed framework a MIMO communication system with Rayleigh fading channel is considered. It is assumed that the channel is changing after every transmitting symbol vector $\mathrm{x}_{\mathrm{Nt}}$ with dimension $\left(\mathrm{N}_{\mathrm{t}} \times 1\right)$ and Binary Phase Shift Keying (BPSK) modulation schemes is considered for simplicity. Figure 2-4 present the comparative results for performance lower bound for $\mathrm{MIMO}$ systems with dimension ranges $d=\{2,4,6,8\}$ for $Z F, M M S E$ and ML detection schemes, respectively. Tightness of the analytical frameworks with respect to actual simulation results were the main focus of this work. The proposed performance lower bound provides tighter lower bound with respect to simulation results as compared to existing framework for receiver with $\mathrm{ZF}$ detection.

At $5 \mathrm{~dB}$ of signal to noise ratio with $\mathrm{M \amalg MO}$ dimension ranges $\mathrm{d}=\{2,4,6,8$,$\} , the proposed$ performance bound is $4 \mathrm{~dB}$ tighter than the existing lower
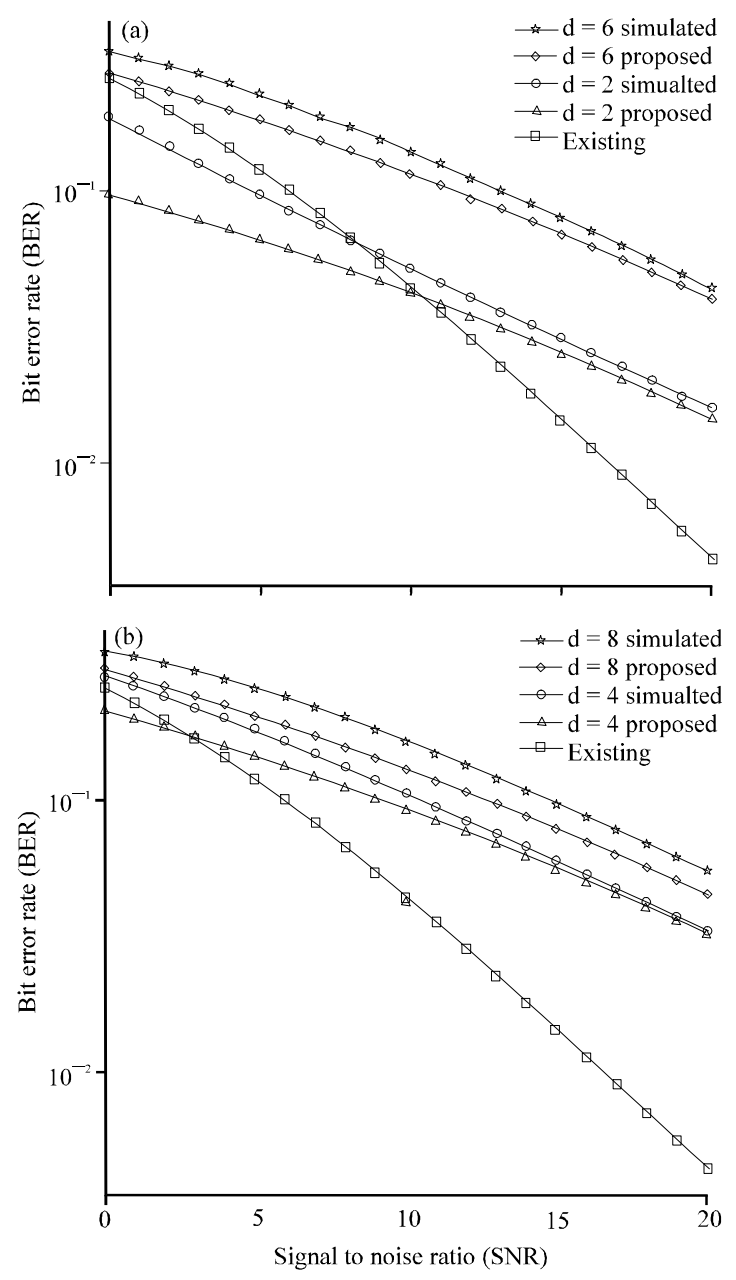

Fig. 2(a-b): Error performance bound comparison for Zero-Forcing (ZF) detection with Multiple-Input Multiple-Output (MIMO) dimension (a) $\mathrm{d}=2$ and $\mathrm{d}=6$ (b) $\mathrm{d}=4$ and $\mathrm{d}=8$

bound as compared to the actual simulated results. At higher signal to noise ratio, the proposed lower bound becomes tighter with respect to actual simulation results.

A comparative study of the existing and proposed analytical frameworks along with actual simulated bit error rate performance for a MIMO receiver with MMSE detector is presented in Fig. 3. As shown in the figure, at $10 \mathrm{~dB}$ of signal to noise ratio the proposed performance bound is $4 \mathrm{~dB}$ tighter than the existing error performance bound in comparison with actual simulated results for $\mathrm{d}=\{2,4,6,8\}$.

Figure 4 presents the performance bound of receiver with $\mathrm{ML}$ detection for $\mathrm{d}=\{2,4,6,8\}$. At $10 \mathrm{~dB}$ of signal to noise ratio, the proposed performance bound for $\mathrm{MLD}$ is 

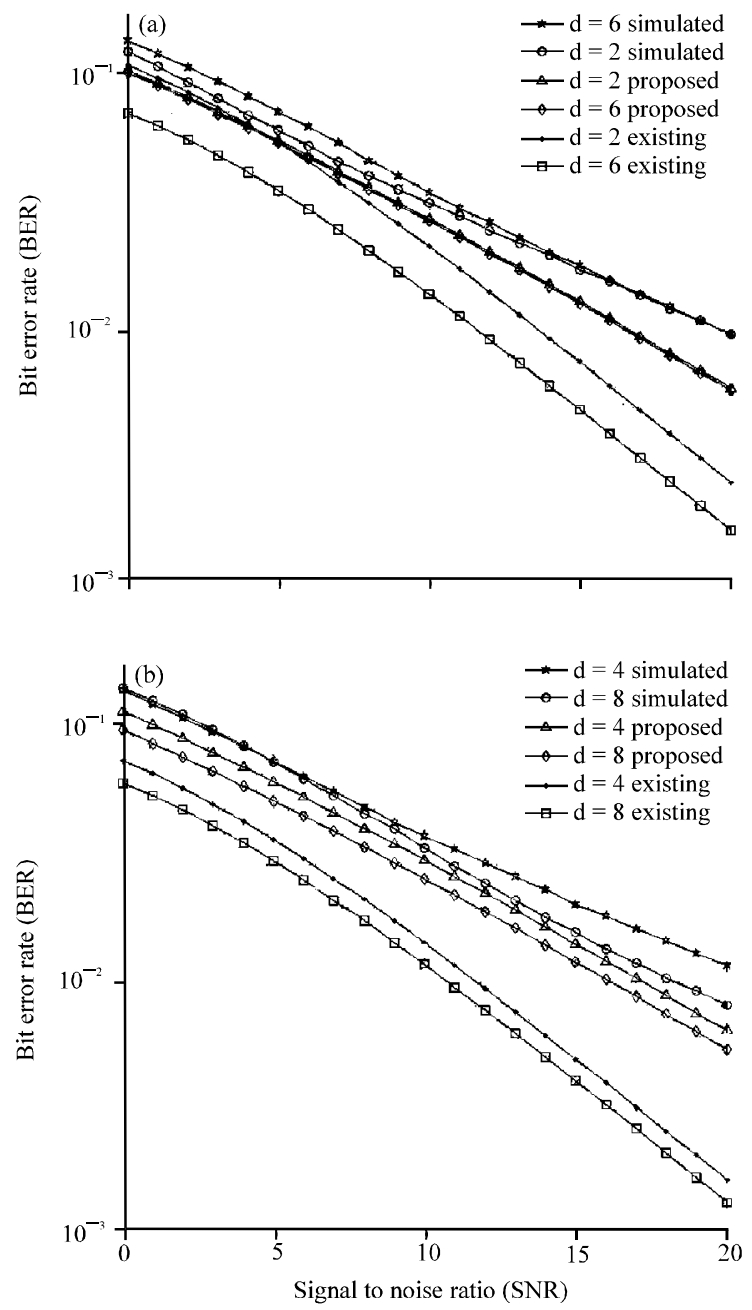

Fig. 3(a-b): Error performance bound comparison for Minimum Mean Square Error (MMSE) detection with Multiple-Input Multiple-Output (MIMO) dimension (a) $d=2$ and $d=6$ (b) $d=4$ and $d=8$

19 and $30 \mathrm{~dB}$ tighter than the existing analytical frameworks for $\mathrm{d}=2$ and $\mathrm{d}=4$, respectively in comparison to actual simulated results.

\section{CONCLUSION}

New analytical frameworks, which provide error performance lower bounds for MIMO system with ZF, MMSE and ML detection schemes have been presented. Tighter approximation have been obtained for the receiver with all three intended detection schemes, in comparison to approximation methods within the existing literature; considering simulated results with respective detection
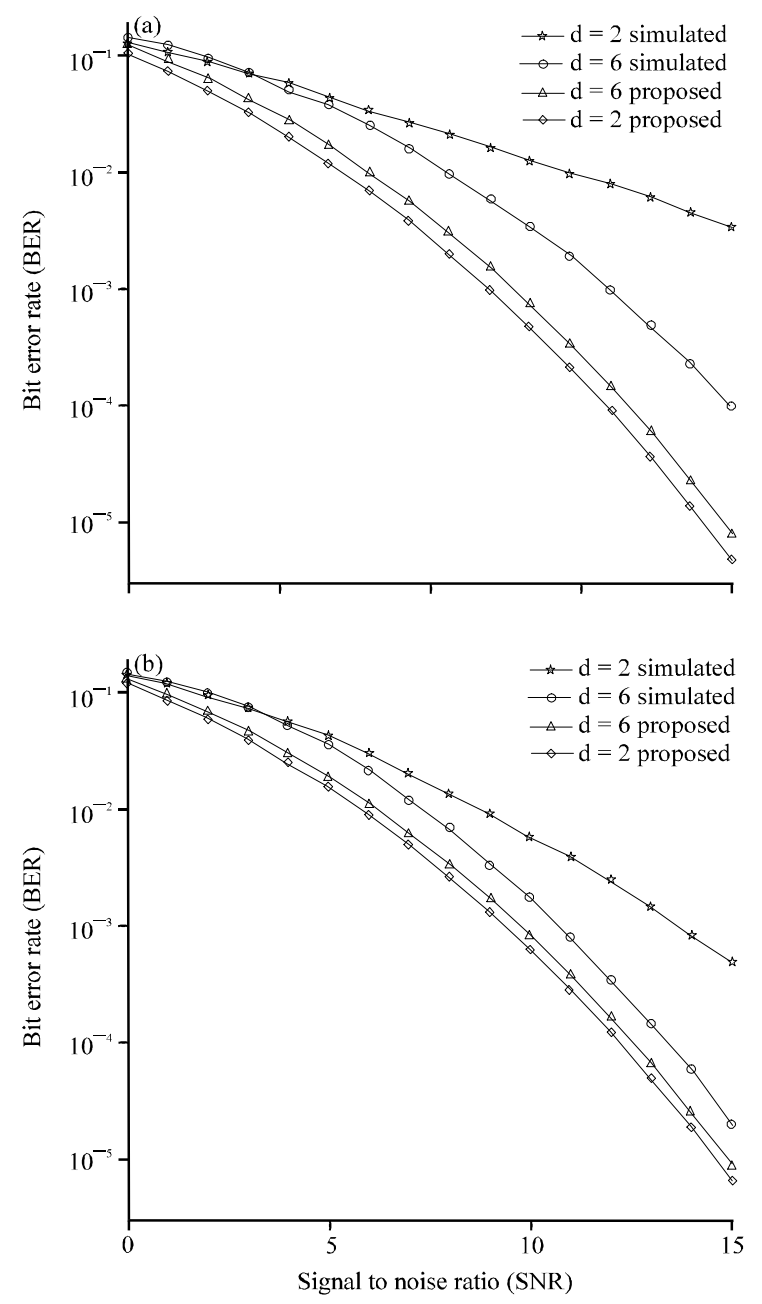

Fig. 4(a-b): Error performance bound comparison for Maximum Likelihood (ML) detection with Multiple-Input Multiple-Output (MIMO) dimension, (a) $\mathrm{d}=2$ and $\mathrm{d}=6$ (b) $\mathrm{d}=4$ and $\mathrm{d}=8$

schemes as reference. The proposed frameworks are expected to be helpful for engineers to approximate the system performance accurately for a symmetric transmitter receiver MIMO communication model. For the future extension of this work, authors are intended to extend this work for asymmetric transmitter receiver MIMO systems.

\section{REFERENCES}

Alamouti, S., 1998. A simple transmit diversity technique for wireless communication. IEEE J. Selecl Areas Commun., 16: 1451-1458. 
Gritsch, G., H. Weinrichter and M. Rupp, 2004. A union bound of the bit error ratio for data transmission over correlated wireless MIMO channels. Proceedings of the IEEE International Conference on Acoustics, Speech and Signal Processing, Volume 4, May 17-21, 2004, IEEE, USA., pp: 405-408.

Guey, J.C., M.P. Fitz, M.R. Bell and W.Y. Kuo, 1999. Signal design for transmitter diversity wireless communication systems over Rayleigh fading channels. IEEE Trans. Communi., 47: 527-537.

Han, J., Q.M. Cui, X.F. Tao and P. Zhang, 2010. SER bound for ordered $\mathrm{ZF}-\mathrm{SIC}$ receiver in M-QAM MIMO system. J. China Univ. Posts Telecommun., 17: 51-55.

Jiang, Y., M.K. Varanasi and J. Li, 2011. Performance analysis of $\mathrm{ZF}$ and MMSE equalizers for $\mathrm{M} M \mathrm{O}$ systems: An in-depth study of the high SNR regime. IEEE Trans. Inform. Theory, 57: 2008-2026.

Kuchi, K. and A.B. Ayyar, 2011. Performance analysis of $\mathrm{ML}$ detection in MIMO systems with co-channel interference. IEEE Communi. Lett., 15: 786-788.

Matthaiou, M., N.D. Chatzidiamantis, G.K. Karagiannidis and J.A. Nossek, 2011. ZF detectors over correlated $\mathrm{K}$ fading $\mathrm{M} M \mathrm{MO}$ channels. IEEE Trans. Communi., 59: 1591-1603.
Molisch, A.F., M. Steinbauer, M. Toeltsch, E. Bonek and R.S. Thoma, 2002. Capacity of MIMO systems based on measured wireless channels. IEEE J. Selected Areas Commun., 20: 561-569.

Molisch, A.F. and M.Z. Win, 2004. MIMO systems with antenna selection. IEEE Microwave Mag., 5: 46-56.

Sanayei, S. and A. Nosratinia, 2004. Antenna selection in MIMO systems. IEEE Communi. Mag., 42: 68-73.

Tarokh, V., N. Seshadri and A.R. Calderbank, 1998. Space-time codes for high data rate wireless communication: Performance criterion and code construction. IEEE Trans. Inform. Theory, 44: 744-765.

Telatar, E., 1999. Capacity of multi-antenna gaussian channels. Eur. Trans. Telecommun., 10: 585-595.

Tse, D. and P. Viswanath, 2005. Fundamentals of Wireless Communication. 1st Edn., Cambridge University Press, Cambridge, UK., ISBN-13: 9780521845274.

Zheng, L. and D.N.C. Tse, 2003. Diversity and multiplexing: A fundamental tradeoff in multiple-antenna channels. IEEE Trans. Inform. Theory, 49: 1073-1096.

Zhu, X. and R.D. Murch, 2002. Performance analysis of maximum likelihood detection in a MIMO antenna system. IEEE Trans. Communi., 50: 187-191. 